\title{
Achten Sie auf drohende Lücken bei Ihrem Versicherungsschutz!
}

\begin{abstract}
Ärzte, die eine Krankentagegeld- und eine Berufsunfähigkeitsversicherung abgeschlossen haben, glauben meist, bei einer längeren Erkrankung zumindest finanziell abgesichert zu sein. Das kann sich aber als Irrtum herausstellen: Versicherungslücken können für böse Überraschungen sorgen.
\end{abstract}

— Viele Ärzte schließen als Schutz bei einer vorübergehenden Arbeitsunfähigkeit eine Krankentagegeldversicherung ab. Kann der Versicherte dauerhaft nicht mehr in seinem Beruf arbeiten, stellen die Unternehmen ihre Leistungen ein.

Meldet sich ein Arzt erst dann beim Berufsunfähigkeitsversicherer oder beim Versorgungswerk, können Monate vergehen, bevor die erste Rentenzahlung auf dem Konto landet. Haben die Versicherten kein finanzielles Polster, kann es in der Wartezeit eng werden.

\section{Leistungen bereits vorsorglich beantragen}

Ärzten, die schon länger schwer krank sind, empfiehlt der Versicherungsjurist Arno Schubach, vorsorglich Leistungen aus der Berufsunfähigkeitsversicherung zu beantragen. „Wenn sich die gesundheitliche Situation bessert, kann man den Antrag problemlos zurückziehen."

In den meisten Fällen ist das Krankentagegeld höher als die Berufsunfähigkeitsdeckung. „Wer versucht, auf das höhere Krankentagegeld zu schielen, erleidet in der Regel Schiffbruch“, warnt Schubach. Zwar zahlt der Berufsunfähigkeitsversicherer gegebenenfalls rückwirkend. „Ist in diesem Zeitraum Krankentagegeld geflossen, kann es sein, dass der Kunde das zurückzahlen muss", sagt der Koblenzer Rechtsanwalt.

Das sei vor allem dann ein Problem, wenn das Tagegeld bereits ausgegeben ist und die Leistung aus der Berufsunfähigkeit geringer ist. Schubach kennt den

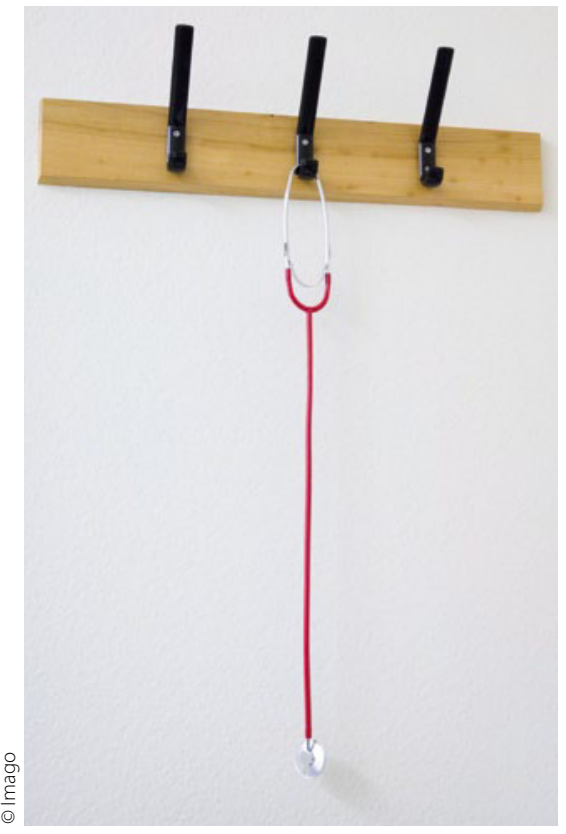

Viele Ärzte sind nicht hinreichend gegen Einnahmeausfälle bei längerer Erkrankung abgesichert.

Fall einer an Krebs erkrankten Frau, die 9000 Euro Krankentagegeld zurückzahlen musste, ihre monatliche Berufsunfähigkeitsrente betrug aber nur 800 Euro.

\section{Ab wann ist ein Arzt berufsunfähig?}

Bei den berufsständischen Versorgungswerken hängt die Latte sehr hoch, bevor ein Mitglied als berufsunfähig eingestuft wird. Die Definition, wann ein Arzt seinen Beruf noch ausüben kann, ist weit gefasst. „Die berufsbezogene völlige Erwerbsunfähigkeit ist eine hohe Hürde", bestätigt Stefan Strunk, Sprecher der Ar- beitsgemeinschaft berufsständischer Versorgungswerke.

Ärzte, die zusätzlich eine private Berufsunfähigkeitsversicherung abgeschlossen haben, sollten abklären, ob der Berufsunfähigkeitsschutz auf die konkrete zuletzt ausgeübte Tätigkeit abzielt. „Es macht einen Unterschied, ob jemand nicht mehr als Arzt tätig sein kann oder nicht mehr in seiner Spezialisierung “, so Schubach. Ärzte sollten prüfen, ob sie eine Gruppenversicherung für die Berufsunfähigkeit abschließen können. Dort sind die Bedingungen zum Teil besser und auf die spezifischen Bedürfnisse der Klientel abgestimmt.

„Es gibt immer noch viele Versicherer, die Kunden auf andere Tätigkeiten verweisen“, sagt Michael Wortberg, Versicherungsexperte bei der Verbraucherzentrale Rheinland-Pfalz. „Bei Altverträgen sollte man mit dem Versicherer über eine Umstellung reden“, rät Jurist Schubach. Beide empfehlen, Krankentagegeld und Berufsunfähigkeit beim selben Anbieter zu versichern. Streitfragen klären die Konzern-Unternehmen dann untereinander und nicht auf dem Rücken der Versicherten.

\section{Abgedeckt sind nur die persönlichen Einnahmen}

Die Krankentagegeldversicherung sei für Ärzte grundsätzlich sinnvoll, sagt Schubach. Allerdings deckt die Police nur die persönlichen Einnahmen des Arztes ab, nicht aber den allgemeinen Kostenapparat, der auch beim Ausfall des Praxisinhabers weiterläuft. Dafür seien Praxisunterbrechungsversicherungen eine sinnvolle Ergänzung.

ILSE SCHLINGENSIEPEN • 